\title{
Protée
}

\section{L'art d'enchaîner}

\section{La fluidité dans le récit contemporain}

\section{Marie-Pascale Huglo}

Volume 34, numéro 2-3, automne-hiver 2006

Actualités du récit. Pratiques, théories, modèles

URI : https://id.erudit.org/iderudit/014271ar

DOI : https://doi.org/10.7202/014271ar

Aller au sommaire du numéro

\section{Éditeur(s)}

Département des arts et lettres - Université du Québec à Chicoutimi

ISSN

0300-3523 (imprimé)

1708-2307 (numérique)

Découvrir la revue

Citer cet article

Huglo, M.-P. (2006). L'art d'enchaîner : la fluidité dans le récit contemporain. Protée, 34(2-3), 127-137. https://doi.org/10.7202/014271ar
Résumé de l'article

Cet article examine de façon exploratoire les modes d'enchaînement dans quelques récits contemporains, afin de repérer un " geste » distinctif parmi la diversité des styles et des pratiques narratives actuelles. C'est donc en un parcours indicatif, dans lequel Renaud Camus côtoie Nancy Huston, Annie Ernaux, Chloé Delaume, Michel Houellebecq et Jean-Philippe Toussaint, que je tente de circonscrire une façon typiquement contemporaine d'enchaîner. Au terme de ce parcours, l'enchaînement fluide d'éléments hétérogènes se démarque comme un mode spécifiquement contemporain qui joue avec les codes syntaxique et romanesque établis et les déplace sensiblement. Cette fluidité des enchaînements n'a rien d'illisible et s'inscrit dans le contexte contemporain du retour à la littérature " transitive ». Sur un plan plus formel, on peut la relier au milieu médiatique dans lequel nous baignons, où la fluidité et l'hétérogénéité des enchaînements sont, globalement, la règle. Les modes d'enchaînements ressortent comme l'un des lieux où l'intermédialité à l'oeuvre dans la littérature se manifeste, cette dernière nous permettant de mieux saisir les transformations du récit contemporain.
Ce document est protégé par la loi sur le droit d'auteur. L'utilisation des services d’Érudit (y compris la reproduction) est assujettie à sa politique d'utilisation que vous pouvez consulter en ligne.

https://apropos.erudit.org/fr/usagers/politique-dutilisation/ 


\section{L'ART D'ENCHAÎNER: LA FLUIDITÉ DANS LE RÉCIT CONTEMPORAIN}

MARIE-PASCALE HuGlo

Parler d'enchaînement à propos du récit renvoie inévitablement aux grammaires narratives issues de l'approche narratologique. L'enchaînement des motifs, des fonctions, des actions ou des séquences a fait l'objet de maintes syntaxes visant à décrire la structure du récit, à en ressaisir la logique et les invariants. Mais parler d'enchaînement peut fort bien renvoyer aux relations entre les éléments de la phrase et du discours, c'est-à-dire à des manifestations de surface dont les effets et les implications narratives sont assez peu pris en compte ${ }^{1}$. En ce sens, les enchaînements qui nous intéressent se situent en deçà des relations logiques, chronologiques ou associatives, dont l'ordre et les désordres sont étroitement associés à la narrativité elle-même. Ce sont des manifestations discursives diverses, des façons de conjoindre dont on peut se demander ce qu'elles impliquent. Il s'agit donc de cerner une façon d'enchaîner caractéristique de la prose narrative d'aujourd'hui. L'approche est délicate: le corpus littéraire est vaste (pour ne pas dire infini) et les modes d'enchaînement sont disséminés à tous les niveaux du récit, ce qui atomise l'analyse. Manifestation diffuse, polymorphe et omniprésente, l'enchainement résiste à la saisie même si l'on en éprouve, plus ou moins distinctement, les effets.

Afin de mieux capter ce qui, dans l'art d'enchainer, se démarque aujourd'hui, je me suis basée sur une impression de lecture réitérée, celle d'une fluidité particulière à plusieurs récits contemporains. Ce sont des manifestations ponctuelles qui, parce qu'elles sont récurrentes, me semblent signaler, dans la façon de nouer les éléments du récit, une transformation sur laquelle j'aimerais me pencher. Autant la discontinuité et l'hybridation narratives ont, jusqu'à ce jour, mobilisé l'attention, autant la fluidité reste comparativement peu étudiée. Or - et c'est là où elle présente un intérêt - la fluidité n'est pas le propre d'un style limpide, qui coule bien, dont les diverses composantes s'articulent sans heurts ${ }^{2}$. Il s'agit plutôt de l'enchaînement continu d'éléments hétérogènes, d'un défilement fluide d'éléments disparates ou disjoints. L'objectif principal de cet article est de mieux circonscrire, de façon exploratoire, la fluidité en question. Je m'efforcerai ensuite d'élargir le propos en cherchant à comprendre globalement ce phénomène. 


\section{FRONTIĖRES}

Dans Syntaxe ou l'autre dans la langue, Renaud Camus s'intéresse aux tournures syntaxiques fautives récemment entrées dans l'usage. "Sur comment" ouvre le propos et devient le symptôme de ce qui pourrait bien être un "univers post-syntaxique» (Camus, 2004: 25). Loin de prendre les fautes de syntaxe devenues monnaie courante à la légère, Renaud Camus les associe à une société gouvernée par la revendication d'être soi-même, dans laquelle le détour par l'Autre (par le "déjà dit avant moi», par le déjà là dont procède la syntaxe) se trouve évacué. L'indifférence pour la syntaxe serait un symptôme du «soi-mêmisme» (ibid.: 38) dans un monde où le "pareil au même» (ibid.: 46) triomphe partout, dans les médias plus qu'ailleurs. Dans des tournures telles que "[o]n s'est beaucoup interrogé chez France-Yaourt sur comment combiner un maximum d'onctuosité avec une authentique culture zéro-calorie» (ibid.: 78), le sens s'appréhende très bien; il n'y a aucune perte à ce niveau. Ce qui se perd, cependant, c'est la «transition entre le mode affirmatif et le mode interrogatif" (ibid.), au bénéfice «d'un aplatissement général de l'expérience et du monde, d'un actif refus de tout ce qui distingue ou sépare, que ce soit dans l'espace ou le temps, dans la volonté ou dans l'être» (ibid.: 79). Sans nécessairement souscrire à la thèse de Renaud Camus qui, non sans humour d'ailleurs, déplore ce qu'il considère comme un appauvrissement, $\mathrm{j}$ 'accorde moi aussi de l'importance à la syntaxe, dont les hiérarchies, les distinctions et les frontières sont, en effet, affaire de société ${ }^{3}$. Or, ce qui me retient, dans cette affaire, c'est que l'usage d'une formule telle que «sur comment» a la capacité de faire disparaître des frontières, d'abolir des distinctions manifestement devenues inutiles. La différence entre les modes affirmatif et interrogatif s'évanouit pour laisser place à la relation entre sujet et prédicat, plus économique, plus directe ${ }^{4}$. On voit donc que s'il y a une perte, comme le constate Renaud Camus, il y a aussi un gain ou, plutôt, un changement de valeur. La formulation économique et directe "vaut mieux» dans un monde où les communications de masse, qui diffusent les informations dans différents médias et les traduisent dans plusieurs langues, ont un impact sur nos usages de la parole ${ }^{5}$. Ne pas se soucier de l'apparition de «sur comment» revient à adopter tacitement les valeurs de la communication de masse au détriment des valeurs de la distinction et de la hiérarchie, valeurs indissociables de certains usages sociaux, mais aussi de perceptions du monde. Car la syntaxe est, à son tour, constitutive de certains modes d'apparaître. Voir le monde en termes de «sujetprédicat» ou en termes de «modes distinctifs" projette donc deux univers qui s'opposent. Enchaîner comme ceci plutôt que comme cela, c'est impliquer des valeurs et mettre en œuvre des rapports qui configurent nos perceptions et sont configurés par elles. Lorsque nous remarquons un nouvel usage, ce n'est donc pas seulement comme une perte qu'il faut l'aborder: il reste à mesurer les changements que cet usage implique, pour le meilleur ou pour le pire (loin de moi l'idée de faire de ces changements l'instrument d'un progrès). Qu'en est-il, alors, de la fluidité?

Dans «Éloge de la honte», le même Renaud Camus donne un exemple de l'attitude sans vergogne caractéristique de la société «soi-mêmiste» qu'il dénonce. Il s'agit d'un petit récit illustrant les conduites banales qui rompent avec «tout partage de l'espace social (autant dire de l'espace tout court)" (2004: 156) dans des «millions d'occurrences si petites [...], que la loi ne saurait être invitée à y mettre son nez [...]» (ibid.: 158):

Et comprendra très bien ce que je veux dire quiconque a tâché de signifier poliment au locataire de l'appartement $d u$ dessus que Skyrock à toute berzingue à deux heures du matin, ce n'était décidément plus possible, et s'est vu signifier sans circonvolutions que si vous ne pouvez pas dormir c'est entièrement votre problème, hein, pas le mien, est-ce que je vais frapper chez vous au milieu de la nuit, moi, et si vous n'êtes pas content vous n'avez qu'à déménager, c'est pas les trous à rats qui manquent à Landerneau. (Ibid.)

Dans ce micro-récit exemplaire, le discours narratif, dont une partie est rapportée sur le mode indirect 
libre ( Skyrock à toute berzingue à deux heures du matin, ce n'était décidément plus possible»), se transforme en un discours que l'on pourrait qualifier, faute de mieux, d'indirect-direct, puisqu'il glisse subrepticement d'un mode ("que si...») à un autre ( $\ldots$...vous ne pouvez pas dormir...»). Les marques formelles de la citation disparaissent, de sorte que la parole de l'autre semble gruger la voix narrative qui, jusque-là, prenait le récit en charge, évacuant au passage les marques du style soutenu propre à l'écrit au profit de marques d'un style "parlé» ${ }^{6}$. La narration de cet incident de voisinage donne très efficacement à saisir l'art de se dégager de toute responsabilité civile en retournant l'offense contre l'offensé; mais, par le glissement vers le style direct et la gradation correspondante des marques oralisées de subjectivité et d'affect, elle donne à sentir, dans le mouvement même du discours, l'envahissement de la parole de l'autre qui, au bout du compte, occupe tout le terrain. Cette sensation d'envahissement va de pair avec une sensation de fluidité: nuls guillemets n'encadrent la réplique, nul point n'en arrête le cours. La chaîne en assonance des «c'est», «est-ce», «et si», "c'est» renforce l'impression d'un flot de paroles ininterrompu balayant tout sur son passage.

La fluidité passe donc, techniquement, par le renversement en cours de route du discours indirect en discours direct et l'effacement des marques typographiques de la parole d'autrui (les guillemets). La prise en charge de ce discours par la voix narrative, qui, au départ, met le récit à distance, disparaît en chemin, d'où l'effet d'un rapprochement qui accompagne le flux de parole. La hiérarchie et le surplomb narratifs sont abolis, la frontière entre les discours (indirect/direct; narratif/non narratif) est franchie dans un flux verbal continu. Nous percevons toutefois clairement qu'il y a passage, renversement, rapprochement: contrairement à l'exemple cité plus haut (France-Yaourt), les frontières ne disparaissent pas complètement et les différences sont maintenues; la transformation du style, des temps verbaux et des embrayeurs signale sans ambiguité au lecteur que l'on est passé à un autre régime de parole, tandis qu'avec «sur comment», la différence entre les modes affirmatif et interrogatif disparaît comme telle, d'où l'impossibilité de percevoir le passage. La fluidité implique l'atténuation des marques distinctives et le glissement, sans pause ni transition, d'un régime discursif à un autre, mais elle n'implique pas l'abolition des différences ni celle des frontières: qu'elles soient franchies sans autre forme de procès ne signifie pas qu'elles ont disparu de l'horizon «d'entente». Le lecteur s'y retrouve sans difficulté, il sait "qui parle», tout en ressentant l'intrusion de cette parole dans le champ de la narration. Cet effet sert à merveille le propos de Renaud Camus: le partage de la parole (l'invasion "sans vergogne» de l'autre) et le partage de l'espace social se recoupent parfaitement. C'est là un effet particulier qu'on ne saurait généraliser, mais qui nous permet de considérer le discours d'autrui comme un lieu d'enchaînement significatif. La facilité avec laquelle nous entrons dans ce type de narration indique enfin qu'il ne s'agit pas d'une pratique avant-gardiste: nous avons déjà assimilé ce mode d'enchaînement, lequel ne compromet en rien la lisibilité du récit.

Renaud Camus montre, jusque dans son style, l'une des grandes caractéristiques du récit qui réside, fondamentalement, dans la relation. Raconter, c'est relater, mettre en contact différents personnages, des séries événementielles divergentes, des temporalités, des espaces, des savoirs et des imaginaires hétérogènes. Le récit est un terrain de rencontres. Mais la rencontre n'est pas seulement racontée, figurée, mise en récit ou commentée. Elle est aussi ce dont procède le récit, ce qui le constitue sensiblement dans sa discursivité même. C'est là que je me situe lorsque je cherche à distinguer une façon contemporaine d'enchaîner. Par ses accélérations spectaculaires et sa réflexion sur la syntaxe, Renaud Camus permet de faire ressortir la spécificité du croisement entre fluidité et contraste, mais il reste encore à donner un petit aperçu de modes d'enchaînement comparables, ne serait-ce que pour montrer qu'il ne s'agit pas là d'un cas isolé. 


\section{APERÇU DE L'ART D'ENCHAÎNER}

\section{DANS LE RÉCIT CONTEMPORAIN}

La pratique romanesque du monologue intérieur nous a habitués à une forme de fluidité liée à l'intériorité. Le "flux de conscience» est une suite verbale de pensées hétérogènes s'enchaînant en souplesse sur le mode associatif, sans souci pour la logique ou pour la cohérence. La fluidité de la pensée relève de la dictée de l'inconscient (pour reprendre la formule surréaliste) qu'elle narrativise et «cadre» au sein du roman. Dans la littérature contemporaine, fluidité et intériorité font encore la paire, mobilisant un horizon d'attente déjà bien établi. Dans Les Variations Goldberg de Nancy Huston, les pensées des auditeurs d'un concert de clavecin sont transcrites en autant de variations fragmentaires qui font entendre la «voix intérieure» de chacun d'eux. Dans ces variations, le discours atteint une certaine fluidité associative sans compromettre pour autant la

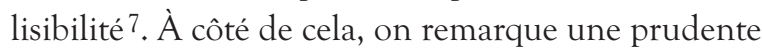
«fluidisation» des discours rapportés; les paroles d'autrui sont, minimalement, signalées par des guillemets, mais elles ne sont pas annoncées:

Suiure. D'à côté. D'en dehors encore une fois. Comme d'habitude. Aider les autres petites filles à s'habiller parce que je ne suis pas assez mignonne pour jouer à la marguerite. "Elle est si habile de ses mains, Adrienne, elle sait si bien coudre déjà».

(Huston, 1984: 20)

L'absence de médiation narrative fluidifie l'intégration du discours d'autrui dans le discours intérieur, mais la frontière entre les deux s'inscrit avec netteté. Ce type de procédé fait de l'intériorité une sorte de voix-radio, capable de citer d'autres voix sans complètement les phagocyter puisque, visiblement, elle les distingue. Nous sommes bien à l'intérieur d'une conscience qui filtre et absorbe toute extériorité sans perdre son identité propre.

Dans Ce qu'ils disent ou rien d'Annie Ernaux, par contre, la frontière entre "moi» et les autres est subtilement brouillée. La narratrice raconte son adolescence dans une prise de parole intime, réflexive, lucide, aux antipodes de la «dictée de l'inconscient»; elle se coupe des autres - en particulier des parents, de la mère -, mais elle est habitée, hantée et même "parlée» par eux:

L'année dernière pourtant je ne pensais qu'à rentrer en seconde $\mathrm{C}$, il faut dire que les profs nous flanquaient la pétoche, juste, très juste, vos notes... Calmes, distingués, mais ça veut dire macache pour $\mathrm{C}$, vous n'avez qu'à être plus intelligents, pas notre faute. À la maison, elle râlait sec, huit en math! c'est pas gras, quand on en met un coup on y arrive. Tu veux finir en usine peut-être? Je sais bien qu'elle a raison, rien à dire contre, si je n'étais pas allée en seconde, couic, le boulot. Tout de même, quand elle me tannait en mars dernier au moment de l'orientation scolaire, je ne l'aimais pas, j'aurais préféré qu'elle ne dise rien. Maintenant, elle est rassurée, pas de pet jusqu'au bac [...]. (Ernaux, 1977: 10)

Le discours des professeurs («juste, très juste, vos notes $\left.{ }^{8}\right)$ et celui d'«elle», la mère («huit en math! [...] Tu veux finir en usine peut-être?»), ne sont pas mis entre guillemets ni introduits par la narratrice de sorte qu'ils se mêlent à sa voix de façon très fluide.

L'intériorité est maintenue dans une réflexivité forte, mais l'adolescente cite, sans le signaler, d'autres voix qui s'opposent à la sienne et qu'elle commente. La narratrice recrée de l'intérieur les conflits et les rapports de force qui la travaillent: il ne s'agit pas d'une joute verbale, comme chez Camus, mais d'un conflit latent où sa voix médite celles des autres qui ont (encore) autorité sur elle. Elle ne leur répond pas «en face»: sa parole est un repli mental, un retranchement, un aprèscoup. L'adolescente fait apparaitre la violence d'autrui (sous ses deux versions professorale et maternelle) dans le surgissement abrupt des discours étrangers: ils «déboulent» sans annonce ni amorce, avec une brutalité que la fluidité rend sensible. L'effet d'intrusion verbale, déjà noté chez Camus, est indissociable ici de l'intériorisation des discours d'autrui.

La narratrice rassemble donc des voix d'abord séparées dans le temps et l'espace au sein d'une même temporalité réflexive, mais son identité est contaminée par les mots étrangers qu'elle convoque. D'une part, la voix adolescente est empruntée (la tournure familière «macache pour $\mathrm{C}$ », aujourd'hui 
tombée en désuétude, le montre bien: elle parle le jargon des adolescents de son âge), d'autre part, elle porte l'empreinte des mots étrangers qu'elle énonce comme s'ils étaient les siens. Qui parle dans «pas de pet jusqu'au bac»: elle ou sa mère? Et qui encore dans "couic, le boulot»? L'absence de marques et d'indications fait ressortir l'intrusion brutale du discours d'autrui, mais elle tend aussi à effacer les frontières entre «moi» et les autres. Autant la narratrice intériorise et commente les mots étrangers, autant ces derniers la contaminent, marquant du même coup l'étrangeté de son propre discours, son «extériorité» même. Là où Huston conforte le cercle intime de chacune des consciences ou des voix, Ernaux le décompose de l'intérieur.

Le brouillage des frontières qui, chez Ernaux, problématise l'intériorité «sur les bords» participe d'un mouvement plus large au sein duquel la fluidité se détache d'une pensée librement associative intérieure - pour rejoindre le flux des discours et des informations remettant l'intériorité en jeu. Dans Le Cri du sablier, Chloé Delaume raconte, dans un style dense au rythme travaillé, un "passé oppressant» (2001: quatrième de couverture). Ses recherches stylistiques et formelles l'amènent à bousculer la syntaxe, à rompre de façon «criante» avec l'usage courant de la langue, mais elle intègre aussi dans son récit des façons de dire et de faire admises dans l'usage. Lire «[c]ar après fiancé et quelques foutraillons elle crut divines sucettes aux fables serties rosaces des mythiques androgynes qui peuvent refusionner" (ibid.: 104), c'est, d'abord, reconnaître un écart syntaxique par rapport à une norme établie. Sans aller jusqu'à l'illisibilité, la phrase fait obstacle à la transparence du sens, elle demande au lecteur un effort soutenu: la compréhension élémentaire bute sur les mots. En revanche, lire, dans l'incipit, «[l]es hommes nombreux forcèrent la porte. Réfugiée au-dedans je ne pouvais qu'entendre. À l'hôpital dit l'un trop tard notèrent les autres" (ibid.: 9), c'est remarquer une certaine étrangeté dans la formulation qui ne fait pas, toutefois, obstacle au sens. La dernière phrase constitue un bon exemple d'enchaînement fluide d'éléments hétérogènes dont l'écart notable «tombe sous le sens». L'effet produit est celui d'un monderadio où les mots circulent de manière diffuse, dans un échange de voix sexuées, anonymes (sans visage). On pourrait dire, ici, que les voix étrangères restent au-dehors sans atteindre la voix narrative qui se tient «au-dedans». Elles constituent un fond sonore sans reprise dialogique ou réflexive. Du même coup, l'autre envahit l'espace narratif: le lecteur apprend qu'une mort a eu lieu, mais il l'apprend, lui aussi, au vol, au détour de ces mots qui ne le visent pas plus qu'ils ne s'adressent au «je». Paroles entrées par effraction, elles s'imposent dans une rumeur dont l'absence de marques distinctives (virgules, guillemets) donne à percevoir la circulation fluide. Ces paroles en l'air impliquent une narrativité et une dramatisation (depuis la découverte d'un ou plusieurs blessé[s] à envoyer d'urgence à l'hôpital jusqu'au constat du «trop tard»), mais le fait qu'elles se suivent presque sans arrêt souligne le contraste entre les deux assertions. Non seulement les deux voix ne sont pas séparées par une virgule, mais le discours direct n'est pas coupé de l'arbitrage narratif («dit l'un [...] notèrent les autres»). L'échange indifférent des voix atteint par là même la narration qui participe du monde-radio où l'hétérogénéité des discours est, d'un même élan, donnée à sentir et neutralisée.

Alors que, chez Renaud Camus, la radio envahissante est l'amorce d'une lutte verbale épique entre le narrateur et le voisin sans vergogne, chez Chloé Delaume, ce sont les autres qui deviennent radio dans une rumeur envahissante, mais sans conflit, sans échange, sans "prise de bec». Chez eux comme chez Ernaux, des seuils sont franchis, la limite entre «moi» et les autres devient poreuse. La fluidité des discours donne à sentir la fragilité des frontières entre les espaces privé et public, l'intérieur et l'extérieur, mais elle permet aussi de percevoir, chez Delaume, l'isolement d'un «je» devenu tout ouie, en retrait des paroles extérieures qui se mêlent et s'échangent dans un flot sonore indifférent.

L'intériorité n'est pas, chez Delaume, évacuée: elle est maintenue comme un repli qui s'énonce non pas 
directement mais dans le rapport second - parfois ludique $^{9}$ - aux mots des autres et dans une capacité à les projeter comme extérieurs. L'absence de confrontation, de dialogue avec "ce qu'ils disent" conforte une posture énonciative en retrait, hors d'atteinte, dont la seule avancée dans le territoire d'autrui se joue au second degré, sur le terrain littéraire de l'écriture isolé du tout venant par son style, sa scansion manifestement à part. L'observatrice, en somme, ne fait pas partie du lot, et c'est en transformant des discours d'autrui en une vaste rumeur extérieure qu'elle conforte sa "posture intérieure», quasi inatteignable, faite de silence et d'écriture.

Les Autres, de Christine Angot, est une vaste orchestration de "ce qu'ils disent». La narratrice met diverses confidences au premier plan, qu'elle fait alterner: on passe sans transition d'un paragraphe à un autre, d'une histoire (d'une voix) à une autre, dans un cycle récurrent qui rythme l'ensemble du récit ${ }^{10}$. La narratrice est la caisse de résonance des mots des autres, elle les interroge et les transcrit dans un flux verbal où discours indirects et directs s'entrelacent étroitement:

Elles éclatent de rire au téléphone, disent qu'elles ne sont pas grandes, un mètre soixante, les cheveux courts. Qu'elles sont en pull et jean. Par exemple étudiantes en droit. Mère célibataire avec deux enfants. Ils sont à l'école. Elles reviennent à leur conversation professionnelle. Alors tu veux qu'on fasse l'amour au téléphone? Il s'agit de ça. (Angot, 2001: 18)

Dans cet extrait, les voix de «elles» sont racontées par un «il» dont le discours est pour ainsi dire réfracté par la voix narrative, celle qui décrète, à la fin, «Il s'agit de ça». Les voix sont ainsi «mixées», et c'est de façon très fluide que l'on passe de la narration ("Elles reviennent à leur conversation professionnelle») au discours direct ("Alors tu veux qu'on fasse l'amour au téléphone?») avec, aussi, des zones d'incertitude ("Ils sont à l'école»). L'imbrication des voix et la fluidité de leur enchainement projettent un «monde-radio» tout en discours, où la part intime, en l'occurrence sexuelle, s'extériorise. Il n'y a plus d'intériorité qui vaille.
Il en va de même - mais tout autrement - avec le premier roman de Houellebecq, Extension du domaine de la lutte, dans lequel le narrateur raconte sa vie (si l'on veut) à la première personne, sans le moindre repli intérieur. Il est l'observateur du monde tel qu'il se dit, tel qu'il se montre, tel qu'il le pense; s'il ne se prive pas de faire remarques, évaluations ou constats, il est lui-même l'objet de ses observations. Il fait donc partie de la rumeur qu'il commente sur le mode d'une longue conversation avec le lecteur, à son tour convié à entrer dans le «domaine de la lutte» (Houellebecq, 1994: 14). Avec Houellebecq comme avec Angot, nous quittons sans l'ombre d'un doute le roman de l'intériorité au sein duquel la fluidité associative ou verbale a trouvé un terrain propice à

l'épanouissement. À dire vrai, Houellebecq joue très peu, comparativement à Ernaux, Delaume ou Angot, sur la fluidité des discours; à l'enchaînement fluide d'éléments narratifs et discursifs, il préfère l'usage des guillemets qui distinguent le discours des autres, le morcelant comme autant d'échantillons de ce qu'ils disent ${ }^{11}$ ou comme des portions (tronquées) de dialogue. Dans un cas comme dans l'autre, le narrateur fait office de «régie» et il en va de même avec les discours rapportés sur le mode indirect ${ }^{12}$. Ces procédés hérités du réalisme ont pour effet d'épingler les citations comme autant de prélèvements du discours social, dont l'exemplarité affichée sape la valorisation romanesque de la subjectivité. Mais à l'usage "extensif» des guillemets et du discours indirect correspondent quelques occurrences où les discours s'enchaînent de façon plus fluide. C'est le cas avec les propos de la réceptionniste rapportés, en principe, sur le mode indirect:

D'après elle, tout le monde devrait se conformer à une

méthodologie rigoureuse basée sur la programmation structurée; et au lieu de ça c'est l'anarchie, les programmes sont écrits n'importe comment, chacun fait ce qu'il veut dans son coin sans s'occuper des autres, il n'y a pas d'entente, il n'y a pas de projet général, il n'y a pas d'harmonie, Paris est une ville atroce, les gens ne se rencontrent pas, ils ne s'intéressent même pas à leur travail, tout est superficiel, chacun rentre chez soi à six heures, travail fini ou pas, tout le monde s'en fout. (Ibid.: 27) 
Le recul narratif propre au discours indirect n'est momentanément plus perceptible. Les phrases semblent s'énoncer toutes seules, et c'est bien de cela qu'il s'agit: d'une quelconque litanie de plaintes qui passent du coq à l'âne comme si de rien n'était (on attendrait un point avant «Paris est une ville atroce», qui déplace l'objet des récriminations). Comme chez Camus, on a là une "sortie» qui évacue tout sur son passage, mais le personnage-narrateur de Houellebecq reste indifférent. C'est, d'ailleurs, comme enchaînement indifférencié d'éléments disparates que la fluidité se manifeste le mieux chez lui, jouant autant de l'entendu que du vu: le monde-télé prend le pas sur le monde-radio. On passe sans crier gare d'éléments d'information caractérisant un «type»ou un échange à des détails anodins:

Il n'arrête pas de parler de fric et de placements [...]. Il compte sur un taux d'augmentation légèrement supérieur à l'inflation. Il me fatigue un peu; je n'arrive pas vraiment à lui répondre. Sa moustache bouge. (Ibid.: 18; je souligne);

Afin d'accréditer l'idée je prononce quelques phrases sur les normes scandinaves et la commutation de réseaux; Schnäbele, sur la défensive, se replie sur sa chaise; je vais me chercher une crème caramel. (Ibid.: 59; je souligne)

L'important et l'accessoire sont mis sur un même plan, sans distinction ni hiérarchie. L'attelage entre l'abstrait et le concret (exemple deux), entre le typique et le contingent (exemple un) envahit l'espace romanesque dont les séquences spatio-temporelles sont discrètement disparates et, pour finir, ne mènent à rien. C'est dans ce type d'enchainement que se déclare «l'articulation plus plate, plus concise, plus morne» (ibid.: 42) qui, selon le narrateur d'Extension $d u$ domaine de la lutte, reste à inventer pour dire l'uniformisation du monde contemporain et l'impossibilité des relations humaines (ibid.: 16).

On retrouve cette indifférenciation du réel dans L'Appareil photo de Jean-Philippe Toussaint. La tonalité de Toussaint est certes plus enjouée que celle de Houellebecq, mais l'indifférence à ce qui arrive et l'étrange lutte qui s'engage contre la «réalité» sont bel et bien à l'œuvre. Pour Michel Biron, ce roman n'a «rien à représenter sinon [...] une succession rapide et désordonnée d'images relevant de la contiguïté du vidéoclip plutôt que de la continuité du long métrage» (1989: 12). Le récit va son train sans que l'on sache où il mène; actions, paysages et discours se suivent sans fil directeur apparent:

Le ciel, que je regardais à l'occasion, nuageux et sans lune, se déplaçait sous le vent dans un tumulte de nuages sombres qui se précipitaient en silence vers d'autres cieux. Le restaurant présentait une enseigne lumineuse et nous avions accès à la salle par une petite porte de jardin, grillagée, qui donnait sur la rue. Sur le perron, que des lumières tamisées éclairaient diffusément, se tenait un maître d'hôtel indien, que je saluai à distance dans l'allée, tout en craignant d'être un peu en avance. Mais pas du tout, pas du tout, et, inclinant son accueillant visage baigné de reflets rouges, il m'apprit d'un air navré que c'était complet. Full, dit-il. Full, dis-je. Full, dit-il. Well, dis-je, et, rebroussant chemin, j'allai m'en ouvrir à Pascale. (Toussaint, 1988: 76)

Ce passage, d'abord très visuel, enchaîne de façon fluide une série de plans (le ciel, le restaurant, le perron) dont les deux premiers sont disjoints. Étrange promenade, en effet, où l'on passe sans transition du ciel au restaurant dans une suite qui, loin de marquer son caractère elliptique, semble naturellement glisser d'un plan à un autre. La temporalité n'est pas non plus dénuée d'étrangeté: à la paradoxale vitesse qui fait de chaque plan visuel une sorte de pause contemplative, sur laquelle on ne s'arrête pourtant pas, correspond la lenteur piétinante du dialogue, dont le caractère accessoire ressort d'autant plus que l'on sait déjà de quoi il retourne (le restaurant est complet). Il est cependant rapporté verbatim, les paroles prononcées faisant écho aux verbes introducteurs dans un combat terme à terme assez comique. Nulle ambiguité n'accompagne ces paroles, on sait parfaitement qui parle, mais l'absence de guillemets comme d'italiques (il s'agit pourtant de mots étrangers) égalise le rapport entre discours direct et code narratif au sein de phrases très brèves qui contrastent avec l'amplitude du passage. À la fluidité saccadée du «dialogue» correspond une fluidité plus douce qui enchaîne des éléments visuels à distance. 
Comme chez Houellebecq, le «je» s'extériorise (dans le combat verbal) et l'accessoire se déploie; comme chez Ernaux, les frontières entre le narrateur et les autres deviennent momentanément poreuses, mais la fluidité fait aussi l'objet, chez Toussaint, d'un jeu de vitesses et de variations dont la relative étrangeté n'est peut-être pas sans rapport avec le monde-télé dont il a déjà été question.

\section{DYNAMIQUE DES FLUIDES}

Au terme de cet aperçu, j'espère avoir fait ressortir l'enchaînement fluide d'éléments disparates comme un trait du récit contemporain. Loin de reposer sur des «charnières» ou sur des «transitions» capables de ménager le passage entre des parties distinctes et séparées, la fluidité contemporaine tend à passer directement, sans transition, d'un élément à un autre et à brouiller, sans les effacer, des frontières distinctives. L'échange verbal (et, tout particulièrement, le rapport qui s'instaure entre le «je» qui raconte et les autres) ressort comme un laboratoire où les manières d'enchaîner contemporaines se déplacent de façon très inventive. Sans chercher à minimiser les différences entre les cas abordés (bien au contraire), je constate que c'est sur le plan des discours rapportés que la fluidité contemporaine se démarque le mieux. En effet, elle se déploie sur le fond d'un code romanesque bien établi, ce qui permet de pointer précisément l'amenuisement des frontières et l'absence de transition. Loin de "ménager» l'écart entre les voix, la fluidité l'accentue (d'où l'effet d'intrusion, de brusquerie, de contamination ou de saturation de la parole) et le rend parfois problématique ("qui parle?», me suis-je demandé ici et là). Il est également apparu que le flux n'est pas, dans le roman contemporain, réservé au discours intérieur supposément libéré des règles de la syntaxe et de la cohérence. La fluidisation, parfois très ponctuelle, des éléments du récit participe plutôt du renouvellement contemporain du réalisme ${ }^{13}$ : si elle passe par un point de vue individuel ou intime, elle se projette comme extérieure, comme mode d'apparition du monde qui déborde les frontières de la conscience ou encore l'envahit, la contamine. Enfin, malgré la particularité de la mise en forme perceptible chez Delaume ou Angot, il semble que la fluidité n'implique pas l'illisibilité, loin de là. On comprend par là que l'égalisation des paroles, des événements ou des descriptions enchaînés presque sans transition participe aujourd'hui de notre horizon d'attente au même titre que la distinction syntaxique plus "articulée»: les distinctions persistent, les marques et les articulations, elles, s'atténuent, se déplacent, disparaissent. La différenciation des éléments du roman (narration/dialogue/description) et la hiérarchie entre l'important et l'accessoire constituent encore des repères, mais ces repères permettent de déplacer des frontières au sein du récit et de jouer avec elles. Le souci de l'enchaînement n'a plus l'élégance classique pour horizon: d'autres façons de faire sont à l'œuvre qui, loin de signaler une perte pure et simple, pointeraient vers autre chose, mais quoi?

On pourrait se demander, par exemple, si la technique du montage qui, à l'ère des ordinateurs personnels, participe à la "fabrique de la prose» autant que la syntaxe n'est pas en partie responsable de ces nouvelles façons de faire qui travaillent des poétiques romanesques très éloignées les unes des autres. La technique du montage est souvent associée, en littérature, à la discontinuité, à la répartition non linéaire du texte. Or, dans le deuxième numéro de la Revue de littérature générale, l'écriture est vue comme un tressage, un montage, un mixage, un couper-coller ou un sampling; elle produit des textes non linéaires, fragmentés, mais aussi continus. Il importe de souligner ce dernier aspect, trop souvent laissé de côté à propos de montage. Pierre Alféri et Olivier Cadiot parlent ainsi de mixage et de techniques mixtes, insistant à la fois sur l'hétérogénéité (des techniques, des matériaux) et sur la fluidité de la prose:

Calculs et fluides : le flux des phrases dégrade et fond plus ou moins les formations denses qu'il déplace. Digestion, c'est-à-dire aussi bien vitesse. Les contours d'un corps étrange dans le texte peuvent s'effacer pour mieux ressortir ensuite.

(Alféri et Cadiot, 1996) 
Pour eux, la fluidité mixte de la littérature est affaire de technique mais aussi de surfaces, car il est temps d'en finir avec la "perspective illusionniste» d'une "profondeur» (ibid.). La mise à plat du texte (ou, peutêtre, sa perception "écranique») coïncide étrangement avec la mise à plat de la réalité qui défile comme une bande sonore ou un film, le défilement lui-même produisant des reliefs, des contrastes, des vitesses et des effets sensibles susceptibles d'intensifier, d'aiguiser notre perception de la platitude ou de la rumeur du monde.

Cela m'amène à revenir, pour conclure, à la question posée par le narrateur de Houellebecq, pour qui technique d'écriture et perception de la réalité sont liées ${ }^{14}$.

Dans l'extériorisation des voix chez Delaume, dans la reprise intérieure d'un dialogue qui n'a pas eu lieu chez Ernaux, dans la nullité du duel verbal chez Toussaint, dans les dialogues tronqués chez Houellebecq, on pourrait voir aussi, en effet, le signe d'une difficulté à échanger, à entrer en relation avec l'autre bien que cet autre soit omniprésent. De la même façon, la succession brutale des discours et des événements, le cours fluide et hasardeux des choses seraient représentatifs de notre train de vie déboussolé, mais pris de vitesse. La contiguité narrative, dont Michel Biron parle à propos de L'Appareil photo de Toussaint, serait à l'image de nos vies sans autorité, suite ininterrompue d'incidents et d'impressions qui s'enchaînent sans logique ni nécessité apparente. Il serait oiseux de prétendre qu'entre les univers désenchantés de Houellebecq, Delaume ou Ernaux et le nôtre, il n'existe aucun rapport: ces romans renouent avec la représentation, ils impliquent des modes et des figures que le lecteur actualise et comprend à sa manière. Des représentations du monde prennent donc forme, entre autres à partir de ces modes d'enchaînement, mais le roman n'est pas pour autant un simple reflet de la réalité. Celle-ci se réfracte à travers un ensemble de relations et de déplacements capables d'atteindre sensiblement le lecteur, de renouveler sa perception du monde dans le monde du récit ${ }^{15}$.
La fluidité des articulations syntaxique et romanesque des éléments du récit pourrait ainsi également relever d'une réfraction esthétique, littéraire, du milieu fluide et hétérogène dans lequel nous baignons. Ce milieu médiatique n'apporte pas seulement un flux incessant d'information et des archives monstrueuses (mais instables), pas seulement un surplus de statistiques, de sites, de données transférables, il apporte aussi une autre façon de concevoir le temps, l'espace, les "connexions». Si nos perceptions sensibles sont bel et bien «appareillées» (Déotte, 2004), c'est-à-dire médiatisées par les dispositifs techniques, on peut considérer que la fluidité hétérogène perçue dans le roman a quelque chose à voir avec des modes d'apparaître contemporains associés aux nouveaux médias qui imprègnent l'écriture et l'univers romanesques. Les termes de monde-radio et de monde-télé mentionnés dans mes analyses pointent en ce sens.

À propos de l' «écriture de montage» (ibid.:297) que serait le cinéma, Jean-Louis Déotte parle d' "hétérogénéité» temporelle (ibid.) et de "fluidité» (ibid.: 311). Dans son éloge du zapping, Hervé Fischer se réjouit de voir que «la succession d'informations non liées" succède "aux vertus, comme aux réductions de la linéarité classique» (2002: 55):

La linéarité reflétait l'ordre du monde, consolidé par une syntaxe et une grammaire strictes et autoritaires. Le zapping fait sauter l'autorité de la grammaire et de la syntaxe, donc la structure, donc la structure sociale et familiale et l'architecture du monde. [...]. Il est la mobilité de l'esprit. (Ibid.: 56)

À cet éloge qui oppose, de façon dichotomique, l'architecture (immobile) de la syntaxe à la mobilité (fluide) du zapping, correspond l'approche de Patrick J. Brunet, plus critique vis-à-vis du mode de pensée favorisé par l'environnement numérique. Dans le passage suivant, le rapport entre la fluidité d'un continuum et l'hétérogénéité fragmentaire est clairement posé:

La praxis de l'Internet (qui ne peut être perçue comme un déroulement linéaire et continu) n'est constituée que de fragments, de ruptures successives, de passages d'un contenu à 
un autre ou d'une interaction à une autre. Des ruptures successives sont provoquées par les passerelles qu'offrent les hyperliens pour passer d'une page d'information à une autre, d'un site Web à un autre ou d'un support écrit à un support imagé ou sonore. Ces passerelles peuvent donner l'illusion de continuité alors qu'il s'agit de ruptures. (2002: 69-70)

Les divers modes d'enchainement liés aux médias contemporains seraient à développer et à différencier, mais il semble que, globalement, nous baignions sans même nous en apercevoir dans des flux hétérogènes avec lesquels nous interagissons sans heurts, sans étonnement, sans conflits (sinon, parfois, de voisinage)... L'enchaînement m'apparaît comme un des lieux où l'intermédialité à l'œuvre au sein de chaque média se manifeste le mieux. Le choix d'aborder la fluidité dans le récit contemporain vient de la conscience de ce milieu intermédial diffus où fluidité et hétérogénéité se croisent. Qu'on le glorifie ou qu'on s'en inquiète, ce milieu imprègne et modifie (in)sensiblement nos façons de percevoir, de penser, d'écrire, d'associer, de raconter. Le "soi-mêmisme" invoqué par Renaud Camus rate cette imprégnation du milieu médiatique qui, volens nolens, appareille nos «visions du monde». Dans la littérature contemporaine, le mode d'apparaître fluide entre en interaction avec d'autres modes dont, bien sûr, l'articulation syntaxique et l'ordre chronologique et causal du récit. Car la «vieille» syntaxe n'a pas encore rendu les armes: elle compose avec ces flux, en joue, les rend d'autant plus sensibles que le roman et l'écriture, qui ont la mémoire longue, résistent aux emportements de la mobilité et de la vitesse. C'est dans ce jeu croisé de forces sous-jacentes que le roman se transforme et forme notre capacité à voir ce dans quoi nous sommes plongés, nous tendant un miroir étrange que nous nous surprenons, encore, à interroger.

\section{NO TES}

1. P. Alféri (1991) et J.-P. Goux (1999) se sont intéressés de près à la syntaxe dans "la fabrique du continu».

2. Les expressions "style limpide", "style fluide", "phrases qui coulent bien (ou mal)» connotent l'aisance. Elles attestent de la valeur communément reconnue à la fluidité comme marque d'élégance dans la continuité. C'est par rapport à cet usage courant que la fluidité, pour ainsi dire heurtée, que je perçois dans les récits contemporains se démarque.

3.P.V. Zima nous rappelle, fort justement, que les débats sur la dissolution de la syntaxe ne datent pas d'aujourd'hui et souligne l'absence de neutralité de la syntaxe: "On pourrait supposer que la structure de la phrase, de la plus grande unité analysée par la linguistique traditionnelle (de Bloomfield), est un phénomène neutre, situé au-delà des antagonismes sociaux et des conflits idéologiques. Or, cette supposition s'avère être fausse lorsqu'on considère les débats et les conflits stylistiques (esthétiques) du passé» (2000, 118). En 1947,

R. Barthes affirmait que « [c]roire à une grammaire unique, pratiquer une langue française pure, c'était prolonger ce fameux mythe de la clarté française dont le destin est si étroitement lié à l'histoire de la France». "Le problème, pour les écrivains d'aujourd'hui, c'est donc de couper l'écriture de ses origines historiques, c'est-à-dire en fait politiques" (2002: 96 et 97).

4. R. Camus propose deux reformulations de l'exemple de FranceYaourt qui sont, effectivement, plus longues, plus complexes: "Au sein de l'entreprise France-Yaourt, nous nous sommes beaucoup interrogés sur ceci : comment concilier la plus grande onctuosité concevable avec notre solide tradition de laitages à très basse teneur calorique? "; "Chez France-Yaourt, la question que se sont posée nos ingénieurs sécurité minceur est celle-ci : comment combiner, etc." (2004: 78-79 et 79).

5. On peut raisonnablement supposer que le souci de France-Yaourt pour l'onctuosité et la basse teneur calorique de ses produits laitiers n'intéresse pas la planète entière, ce qui n'enlève rien à la valeur accordée à la parole simple, directe et économique dans la société contemporaine.

6. Notons que le style soutenu de la voix narrative qui encadre le début du récit avec toute la distance requise est déjà contaminé par une forme d'oralité plus relâchée et porteuse d'émotion : «à toute berzingue" est une locution indubitablement familière qui marque l'excès avec une emphase que le changement de registre répercute - le narrateur lui-même ne se montre-t-il pas, dans cet écart de langage, quelque peu excessif?

7. On retrouve le même principe dans le monologue intérieur de Molly Bloom, qui dérive de façon fluide, sans aucun signe de ponctuation, d'une "pensée " à une autre (Joyce, 1986: 608-644). Dans ce cas, nous sommes à la limite du lisible, la fluidité de l'écriture non ponctuée faisant, paradoxalement, obstacle à la lecture fluide du passage. Chez N. Huston, ce type de jeu avec la ponctuation reste localisé et évite l'illisibilité: «Elle a entendu parler de slips chauffants que les mecs peuvent porter pour se faire cuire les couilles, franchement comment veut-elle qu'on bande avec des trucs comme ça et si on veut baiser le matin il faudrait que je mette le slip d'abord et 
pour combien de temps est-ce que c'est comme un œuf à la coque ou un œuf mollet non mais ça va pas non?»(1984: 29).

8. Il faut distinguer cette citation du passage suivant, que l'on attribue également aux profs : "vous n'avez qu'à être plus intelligents, pas notre faute». Ces paroles n'ont vraisemblablement pas été prononcées. Il s'agirait plutôt de la traduction intérieure de ce que les profs auraient seulement donné à entendre. L'absence d'indication et de marques fragilise, on le voit, la frontière entre les paroles qui auraient été effectivement prononcées et celles qui traduiraient une simple attitude. 9.Voici un exemple: «J'aimerais lui clouer le bec dit-elle en s'approchant l'oreiller à la main. C'est ainsi que la mère nomma Chloé la fille de l'aume parce qu'il est quand même grand temps de se décider Madame dit le pédiatre reprenez donc un Temesta " (Delaume, 2001 : 29). Dans ce passage, la narratrice met celle qu'elle appelle "l'enfant " à distance d'elle-même. Alors qu'elle raconte comment «la mère " lui trouva son nom, elle se baptise dans et par l'écriture en jouant sur les mots. Elle se donne un nom en retournant contre la mère le désir de meurtre, renversant le sens des paroles prononcées en miroir sonore, faisant advenir Chloé, clouant le bec à la mère. À ce miroir sonore ludique et créateur correspond la ligne de fuite des mots des autres dont le flux mêlé est assez contrasté ici. On retrouve la «fluidité heurtée " de la narration et du discours rapporté, l'absence de ponctuation et de marquage, mais le dernier segment ("reprenez donc un Temesta») relève du coq à l'âne. En ce sens, l'hétérogénéité des éléments du discours mis sur un même plan se double d'une hétérogénéité des éléments de sens placés les uns à la suite des autres (quel est le rapport entre la nécessité de se décider à baptiser l'enfant et celle de prendre un autre comprimé?) dans une enfilade discursive et narrative non dénuée d'humour.

10. Je n'inclus pas le montage des voix dans cette réflexion sur l'enchaînement, dans la mesure où la série prend assez rapidement le dessus sur la succession hétérogène: c'est le retour régulé et attendu de telle ou telle voix qui s'impose une fois passée la désorientation initiale. Ce montage régulé de voix hétérogènes n'est bien sûr pas sans rapport avec la fluidité, mais il mobilise d'autres forces - celle de la série, notamment - plus déterminantes.

11. "Il a aussi une pochette qui dépasse de sa veste, plutôt dans le style "voyage sur la planète Mars", et une cravate assortie. Tout son habillement évoque le personnage du cadre commercial hyper dynamique, ne manquant pas d'humour. Quant à moi je suis vêtu d'une parka matelassée et d'un gros pull style "week-end aux Hébrides". J'imagine que dans le jeu de rôles qui est en train de se mettre en place je représenterai l'"homme système", le technicien compétent mais un peu bourru, n'ayant pas le temps de s'occuper de son habillement, et foncièrement incapable de dialoguer avec l'utilisateur" (Houellebecq, 1994: 53).

12. L'exemple suivant propose un mélange de portions de dialogue et de discours indirect: " $\grave{A}$ midi, nous sommes interrompus par une sonnerie stridente et désagréable. Schnäbele ondule vers nous :"On mange ensemble?..." C'est pratiquement sans réplique. Il nous déclare qu'il a quelques petites choses à faire avant le repas, il s'en excuse. Mais nous pouvons venir avec lui, comme ça il nous fera "visiter la maison".
Il nous entraîne dans les couloirs; son acolyte nous suit, deux pas derrière. Tisserand réussit à me glisser qu'il aurait "préféré manger avec les deux minettes du troisième rang" "(Houellebecq, 1994: 56).

13. D. Viart parle d'un "retour à la littérature "transitive" ", caractérisé par un retour au récit, au réel, à l'expression de soi et à l'imagination (2001: 319 et 322).

14. On se souvient que, pour le narrateur d'Extension du domaine de la lutte, "l'articulation plus plate, plus concise, plus morne»

(Houellebecq, 1994: 42) qui reste à inventer est indissociable des relations humaines impossibles et de l'uniformisation du monde contemporain (ibid. : 16).

15. Je renvoie ici à la thèse bien connue des formalistes russes sur la défamiliarisation. Voir à ce propos l'article de C. Ginzburg sur l'estrangement (2001).

\section{RÉFÉREN CES BIBLIO G RAPHIO UES}

AlfÉRI, P. [1991]: Chercher une phrase, Paris, Christian Bourgois. AlfÉRI, P. et O. CADIOT [1996]: "Digest ", Revue de littérature générale, no 2, Paris, P.O.L., n.p.

Angot, C. [2001]: Les Autres, Paris, Stock.

BARTHES, R. [2002]: «Responsabilité de la grammaire», CEuvres complètes, t. 1 (1942-1961), Paris, Seuil, 96-98.

BIRON, M. [1989]: "Fatiguer la réalité”, L'Appareil photo de JeanPhilippe Toussaint", Spirale, n 87, avril, 12. BRUNET, P.J. [2002] : «Internet et la culture de la rupture», dans C. Vandendorpe et D. Bachand (dir.), Hypertextes. Espaces virtuels de lecture et d'écriture, Québec, Nota bene, 61-74.

CAMUS, R. [2004]: Syntaxe ou l'autre dans la langue, Paris, P.O.L. Delaume, C. [2001]: Le Cri du sablier, Tours, Farrago.

DÉOtTe, J.-L. [2004]: L'Époque des appareils, Paris, Lignes \& Manifestes. ERnAUX, A. [1977] : Ce qu'ils disent ou rien et autres conférences, Paris, Gallimard.

FISCHER, H. [2002]: «De la connaissance en arabesque. Éloge du zapping ", dans C. Vandendorpe et D. Bachand (dir.), Hypertextes. Espaces virtuels de lecture et d'écriture, Québec, Nota bene, 55-60. GinzBurg, C. [2001]: «L'estrangement. Préhistoire d'un procédé littéraire ", À distance. Neuf essais sur le point de vue en histoire, Paris, Gallimard, 15-36.

GOUX, J.-P. [1999]: La Fabrique du continu. Essai sur la prose, Seyssel, Champ Vallon.

HouellebecQ, M. [1994]: Extension du domaine de la lutte, Paris, Maurice Nadeau.

Huston, N. [(1981) 1984]: Les Variations Goldberg, romance, Arles, Actes Sud, coll. "Babel».

JOYCE, J. [(1922) 1986]: Ulysses, Londres, Penguin Books.

ToussainT, J.-P. [1988]: L’Appareil photo, Paris, Minuit.

VIART, D. [2001]: «Écrire au présent: l'esthétique contemporaine»,

dans M. Touret et F. Dugast-Portes (dir.), Le Temps des lettres. Quelles périodisations pour l'histoire de la littérature française du 20e siècle?, Rennes, Presses universitaires de Rennes, 317-336.

ZIMA, P.V. [(1985) 2000]: Manuel de sociocritique, Paris, L'Harmattan. 